\title{
IMPACTS OF THE SYMBIOSIS BETWEEN TRICHOMONAS VAGINALIS AND MYCOPLASMA HOMINIS ON THEIR RESPECTIVE DRUG SUSCEPTIBILITIES
}

\section{Ursula Fürnkranz'1,2, Julia Walochnik¹, Birgit Henrich²}

${ }_{1}^{1}$ Institute for Specific Prophylaxis and Tropical Medicine; Medical University of Vienna Kinderspitalgasse 15; 1090 Vienna

${ }_{2}^{2}$ Institute of Medical Microbiology and Hospital Hygiene; Heinrich-Heine-University; Universitätsstraße 1; 40225 Düsseldorf

Background: Screening studies revealed that in some regions up to $90 \%$ of the T. vaginalis isolates are naturally infected with $M$. hominis. This symbiosis has been investigated with regard to its pathogenicity and immunology in the past years. The influence of this symbiosis on the drug resistance of $T$. vaginalis to metronidazole has been discussed controversially, the influence on $M$. hominis drug susceptibility has not been investigated so far.

The aims of the presented study were to evaluate the impact of natural and artificial symbiosis on the drug-susceptibility of $T$. vaginalis to metronidazole and $M$. hominis to gentamicin, ciprofloxacin, moxifloxacin and clindamycin. Moreover, RNA expression levels of genes associated with metronidazole resistance in $T$. vaginalis and mutations in the quinolone resistance determining regions (QRDR) in M. hominis were investigated.

\section{Material and Methods}

\begin{tabular}{|c|c|c|}
\hline $\begin{array}{l}\text { T. vaginalis } \\
\text { uninfected }\end{array}$ & $\begin{array}{l}\text { Naturally infected with } \\
\text { M. hominis }\end{array}$ & $\begin{array}{l}\text { Artificially infected with } \\
\text { M. hominis SS25 }\end{array}$ \\
\hline TVSS25 - & TVSS25+ & TVSS25-MhSS25 \\
\hline TVSS10- & TVSS10+ & n.d. \\
\hline G3 (type strain) & - & G3-MhSS25 \\
\hline
\end{tabular}

TVSS25+: treatment with plasmocin for 2 weeks $\rightarrow$ M. hominis-free TVSS25TVSS10+: treatment with plasmocin for 2 weeks $\rightarrow$ M. hominis-free TVSS10TVSS25+ in M. hominis culture medium (PPLOa) $\rightarrow$ MhSS25 in pure culture

Artificial infection:

$10^{4}$ TVSS25-/G3 daily boostered with $10^{7 / 8} \mathrm{MhSS} 25 / \mathrm{ml}$ for 4 days. Further culture up to 35 days in T. vaginalis culture medium (TYM).

Drug screening: minimal inhibitory concentration (MIC) assay: Metronidazole $0.5 \mu \mathrm{g} / \mathrm{ml}-512 \mu \mathrm{g} / \mathrm{ml}$ in 96 -well plate plus $10^{5} T$. vaginalis $( \pm M$. hominis) $/ \mathrm{ml} 37^{\circ} \mathrm{C}, 48 \mathrm{~h} . \mathrm{MIC}=$ concentration with no moving (=living) $T$. vaginalis examined microscopically

\section{RNA-extraction, qPCR:}

RNA-extraction according to RNeasy Kit. cDNA convertion according to Invitrogen's instructions.

qPCR for flavin reductase 1 [Leitsch D et al., 2014], PFOR [Maed JR et al., 2006]

and ferredoxin [in house design by B. Henrich]; ß-tubulin [Schirm J et al., 2007] as reference gene

\section{M. hominis isolates:}

V475 (from an asymptomatic woman)

MhSS10 (from TVSS10+ - see above)

AKH136 (from the placenta of a woman with preterm delivery)

Co-culture with TVIR78 (ATCC_50138):

see arrificial infection above

\section{M. hominis-release:}

After two weeks: co-culture in PPLOa $\rightarrow$ passaged $M$. hominis in pure culture

\section{Drug screening; Colour changing units, MIC [CLSI, 2011]:}

96-well plates seeded with $180 \mu \mathrm{l}$ of the respective drug solution $(1 \mathrm{mg} / \mathrm{ml})$ in PPLOa. serial 1:10 dilution from $1 \mathrm{mg} / \mathrm{ml}$ to $1 \mu \mathrm{g} / \mathrm{ml} ;+20$ $\mu 1$ of the respective $M$. hominis culture (original [PPLOa] and passaged [p IR78]); $37^{\circ} \mathrm{C}, 48 \mathrm{~h}$. Colour change at $10^{5} \mathrm{M}$. hominis $/ \mathrm{ml}=\mathrm{MIC}$

\section{Amplification and sequencing of ORDRs:}

Amplification of QRDR of gyrA, gyrB, parC and parE was conducted as described [Bébéar CM et al., 1988].

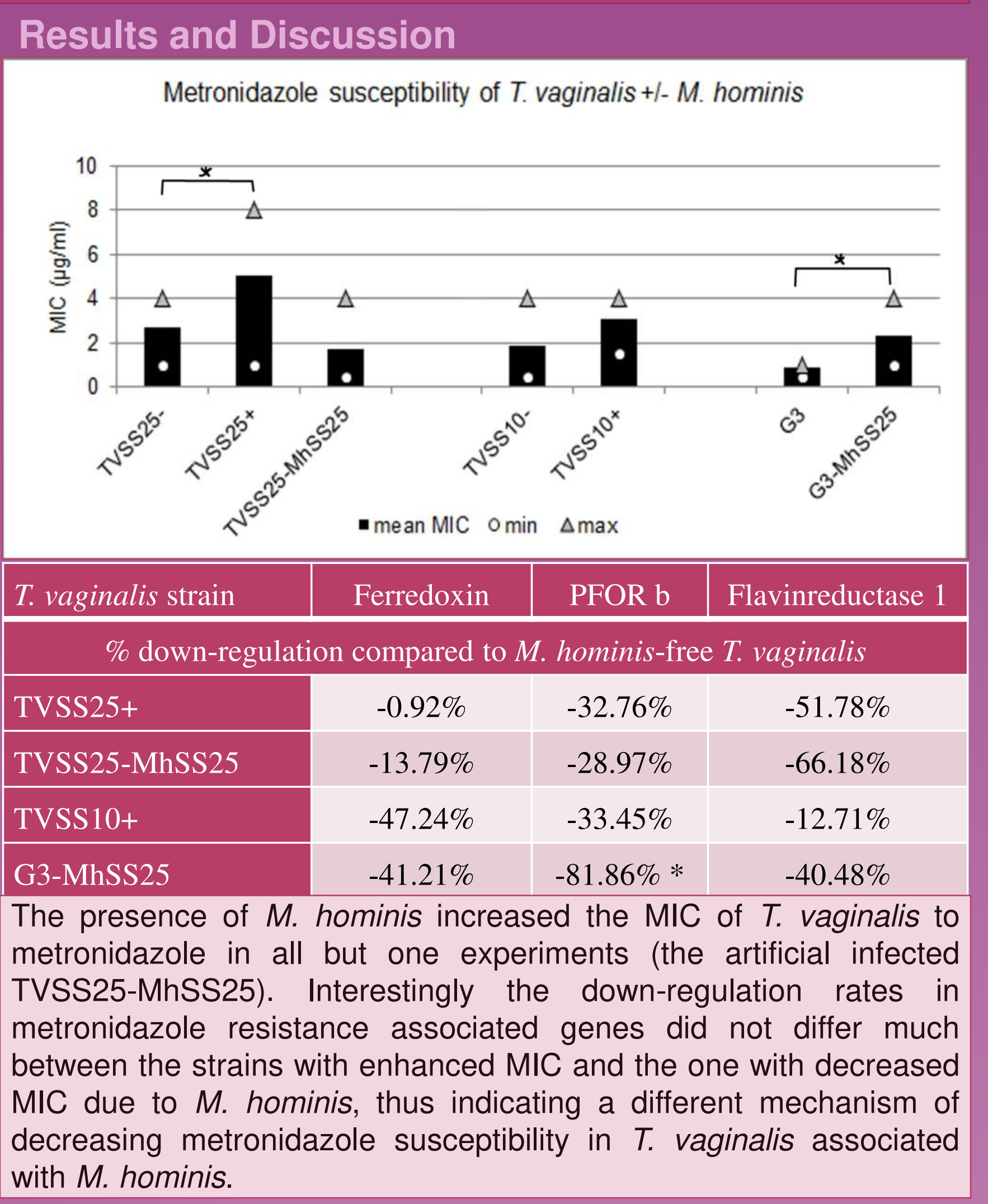

\begin{tabular}{|l|c|c|c|c|}
\hline \multirow{2}{*}{} & \multicolumn{4}{|c|}{ Mean MIC $(\mu \mathrm{g} / \mathrm{ml}) \pm$ mean deviation } \\
\cline { 2 - 5 } & gentamicin & clindamycin & moxifloxacin & ciprofloxacin \\
\hline V475 PPLOa & $2.67 \pm 0.88$ & $2.20 \pm 1.3$ & $1.35 \pm 0.52$ & $2.53 \pm 1.2$ \\
\hline V475 p IR78 & $9.57 \pm 4.7 *$ & $2.36 \pm 0.54$ & $2.50 \pm 0.7 *$ & $2.80 \pm 0.89$ \\
\hline MhSS10 PPLOa & $14.4 \pm 2.56$ & $1.60 \pm 0.96$ & $2.12 \pm 1.18$ & $1.95 \pm 0.45$ \\
\hline MhSS10 p IR78 & $13.30 \pm 6.6$ & $2.00 \pm 0.53$ & $2.40 \pm 1.2$ & $4.23 \pm 2.73$ \\
\hline AKH136 PPLOa & $5.25 \pm 2.75$ & $2.73 \pm 1.26$ & $2.9 \pm 1.3$ & $3.80 \pm 0.7$ \\
\hline AKH136 p IR78 & $3.1 \pm 1.8$ & $2.70 \pm 1.7$ & $1.9 \pm 0$ & $2.08 \pm 1.21$ \\
\hline
\end{tabular}

\begin{tabular}{|l|c|}
\hline Gene & $\begin{array}{l}\text { Mutation in V475 due to passaging } \\
\text { through TVIR78 }\end{array}$ \\
\hline gyrA & (TAT)Y129Y(TAC) \\
& (CAT)H150H(CAC) \\
& (TCA)S153L(TTA) \\
\hline gyrB & (GGT)G449G(GGA) \\
& (GCG)A467A(GCA) \\
& (TTG) L476L(TTA) \\
parC & (AAA)K144R(AGA) \\
& (GAG)E95G(GGG)
\end{tabular}

MIC to all drugs was enhanced due to passaging through TVIR78, except for $M$. hominis strain AKH136. Mutations in the QRDRs of V475 were observed after passaging; mutations in bold leading to amino

acid substitutions, the others remaining silent mutations. In MhSS10 and AKH136 no mutations were observed upon passaging through TVIR78. 